\title{
Design of temperature acquisition and servo debugging board
}

\author{
Sun $\mathrm{Yi}^{1}$, Quanyu Sun ${ }^{1, *}$, and Guowei Shao ${ }^{1}$ \\ ${ }^{1}$ Jiangsu Automation Research Institute, Lianyungang, Jiangsu, 222000, China
}

\begin{abstract}
The temperature acquisition and servo debugging board is used in Flight control computer, mainly for temperature acquisition and servo debugging. Its good functional performance and reliability have a good application prospect.
\end{abstract}

\section{Introduction}

Flight control computer is a computer for the application of flight control system. Its main task is to complete control rate calculation, redundancy management and inflight self-test[1].

The temperature acquisition and servo debugging board is used in Flight control computer, mainly for temperature acquisition and servo debugging.

\section{Principle}

Temperature acquisition and servo debugging board debugging are composed of two functions: temperature acquisition and servo debugging board. The temperature acquisition board collects analog signals input by 4 thermocouples and 4 thermal resistance, converts them into digital quantities and calculates the current temperature values, which are transmitted to the upper computer through CAN bus and temperature debugging serial port. The servo board debug function realizes the 12 way servo debugging serial port and the whole machine debugging serial port's 12 selected 1 functions. The upper computer sends instructions or switch switches, selects the whole machine to debug the serial port and which servo debug serial port to connect, and feedback the serial port which is debugged through the can bus. The specific principle block diagram is shown in Figure 1.

\section{Function}

\subsection{Single Chip Micyoco}

Stm32f103zte6 of STM32 series of ST company is selected as MCU.Stm32f103zet6 integrates abundant peripheral interfaces, $512 \mathrm{k}$ byte flash program memory and $64 \mathrm{~K}$ byte SRAM. With parallel LCD interface, compatible with $8080 / 6800$ mode. Power supply voltage and IO voltage support 2.0-3.6v, embedded with $8 \mathrm{MHz}$, $40 \mathrm{KHz}, 32 \mathrm{kHz} \mathrm{RC}$ oscillator. It has 312 bit $\mathrm{AD}$ converters, conversion range $0-3.6 \mathrm{v}, 2$-channel 12 bit DA converter. It has 12 channel DMA controller and supports timer, SDIO, I2C, SPI and other peripherals. It has 11 timers and up to 13 communication channels:2 I2C interfaces (supporting SMBus/PMBus),5 USART interfaces (supporting ISO7816, Lin, IrDA interfaces and modulation and demodulation control),3 SPI interfaces $(18 \mathrm{~m} \mathrm{bit} / \mathrm{s}), 2$ reusable I2C interfaces, 1 can interface (2.0B active), USB 2.0 full speed interface and SDIO interface

\subsection{External communication interface}

a) One isolated can bus interface;

b) One RS232 communication interface;

c) RS232 servo debugging interface of 1 out of 12;

\subsection{8-way temperature acquisition function}

The temperature acquisition board collects the analog signals of 4 thermocouples and 4 thermistors, converts them into digital values and calculates the current temperature value. 


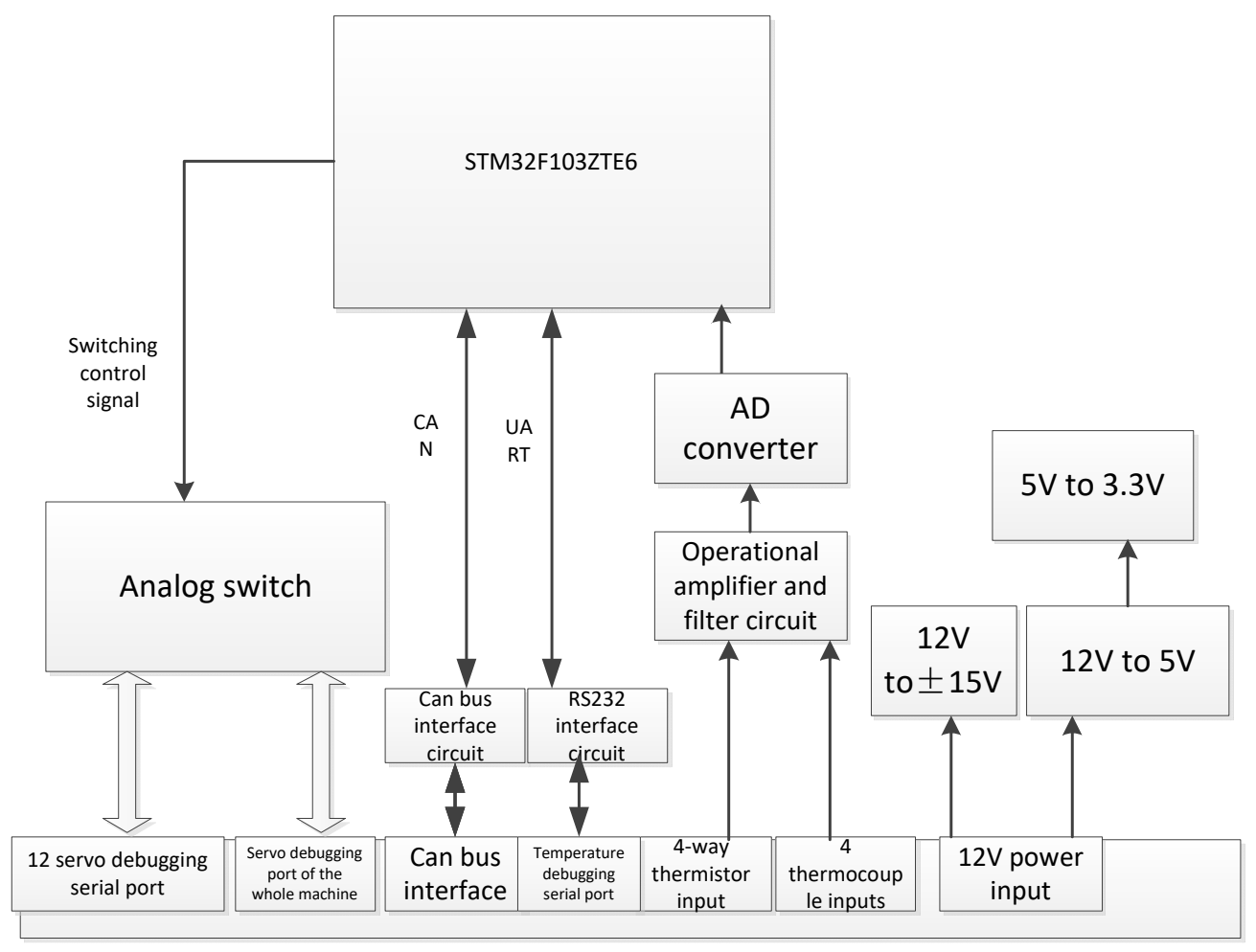

Fig. 1. Principle block diagram.

\subsection{Switching function}

The 12 servo debug serial port and the whole machine debug the serial port's 12 selected 1 functions. The upper computer sends instructions or switch switches, selects the whole machine to debug the serial port and which servo debug serial port to connect, and feedback the serial port which is debugged through the CAN bus.

\section{Hardware design}

\subsection{STM32 Circuit}

Stm32f103zet6 is a high performance processor based on cortex-m3 core. It can work at $72 \mathrm{MHz}$ maximum frequency, and can reach $1.25 \mathrm{DMips} / \mathrm{MHz}$ (Dhrytone 2.1)when the memory is accessed for 0 waiting period. Cortex-m3 is a 32-bit processor kernel. The internal data path is 32-bit, the register is 32-bit, and the memory interface is 32-bit.CM3 adopts Harvard structure, and has independent instruction bus and data bus, which can make the index and data access go hand in hand. In this way, data access no longer occupies instruction bus, thus improving performance. To achieve this feature, $\mathrm{cm} 3$ contains several bus interfaces, each optimized for its own application, and they can work in parallel. On the other hand, the instruction bus and data bus share the same memory space (a unified memory system), and Figure 2 is a simplified view of cortex-m3.

Stm32f103zet6 has 5-way UART interface and 1way can bus interface. In this design, stm32f103zet6 is used to realize the CAN bus, UART and AD acquisition and communication functions of the module. The specific implementation principle is shown in Figure 3.

\subsection{Input analog signal control circuit}

The multi-channel analog signal input selection circuit is composed of an 8-out-of-1 analog switch max307, which completes single ended 8-way input and module self-test input. The voltage input range can reach $-17 \mathrm{~V} \sim+17 \mathrm{~V}$, meeting the signal input range. The selected A/D conversion chip has $0 \sim 5 \mathrm{~V}$, shown in Figure 4.

\subsection{DC / DC isolated power supple}

In this design, the ad switch and RS232 switch need to use $\pm 15 \mathrm{~V}$ power supply, so $\pm 15 \mathrm{~V}$ power supply module is required in this scheme. In the scheme, the $6 \mathrm{~W} \mathrm{dc} / \mathrm{dc}$ DC conversion module VFA of Guangzhou jinshengyang Technology Co., Ltd. is selected_Mp-6w: isolation voltage $>1500 \mathrm{vdc}, \mathrm{MTBF}>1000000$ hours, high voltage stability, low ripple, wide temperature working conditions, the specific selection model vfa1215mp-6w: output $\pm 15 \mathrm{~V}$ two-stage voltage, current $\pm 200 \mathrm{~mA}$. 


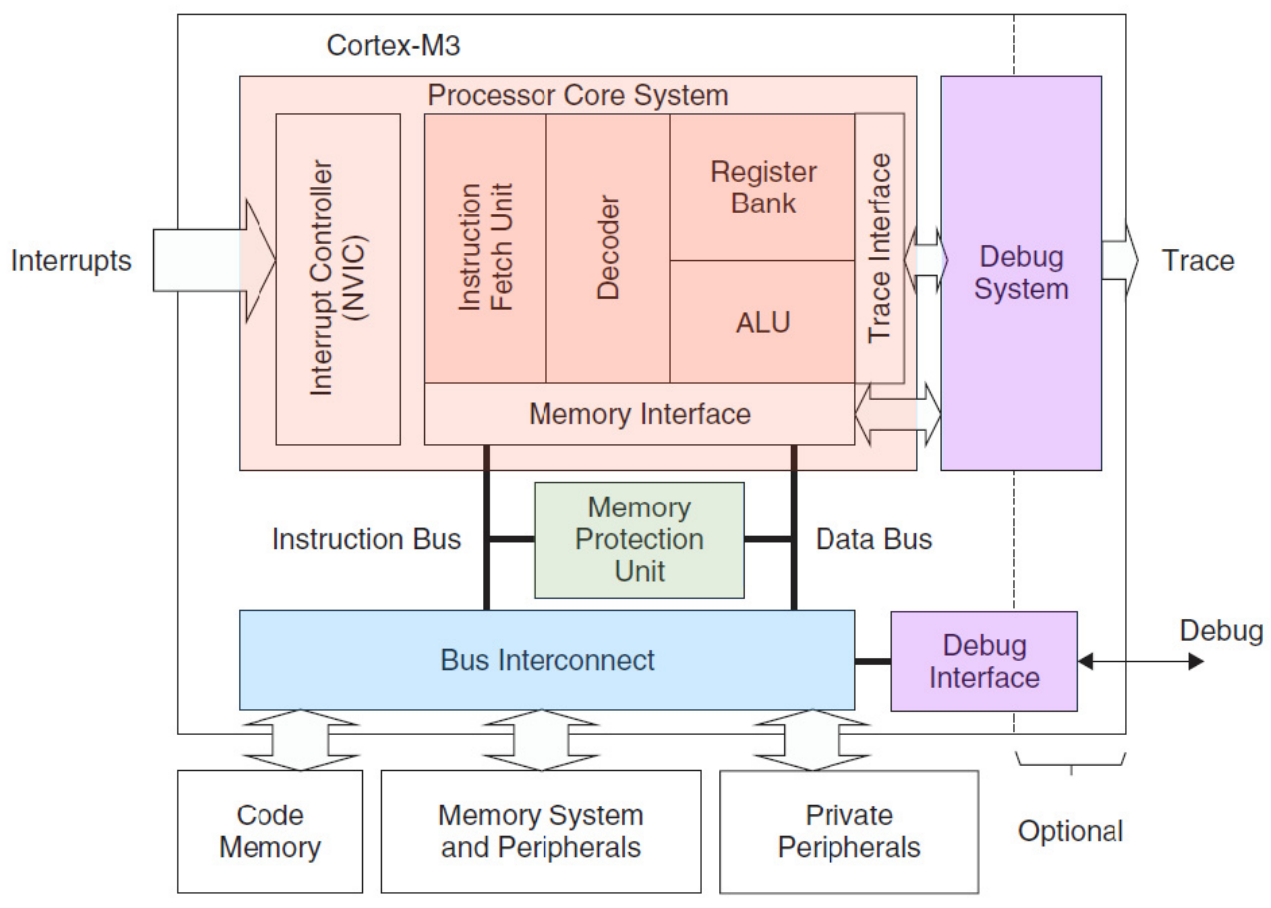

Fig. 2. Simplified view of cortex - M3

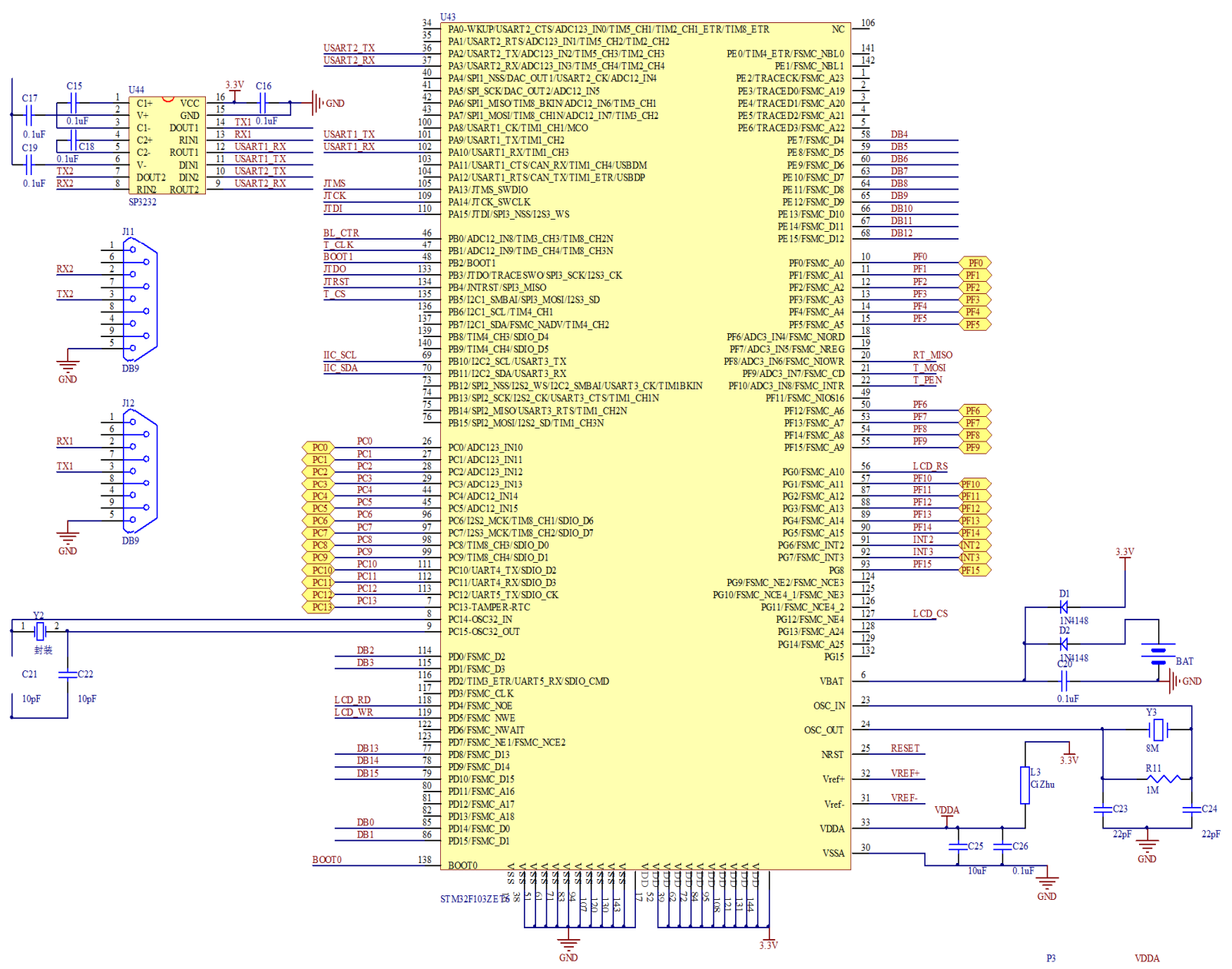

Fig. 3. STM32 Circuit. 


\subsection{Switching circuit}

The 12 channel RS232 switching circuit is composed of two 16 choose one analog switches max306 to complete the switching of 12 channel RX and TX signal input and output. The voltage input range can reach $-17 \mathrm{~V} \sim+17 \mathrm{~V}$, meeting the RS232 signal input range.

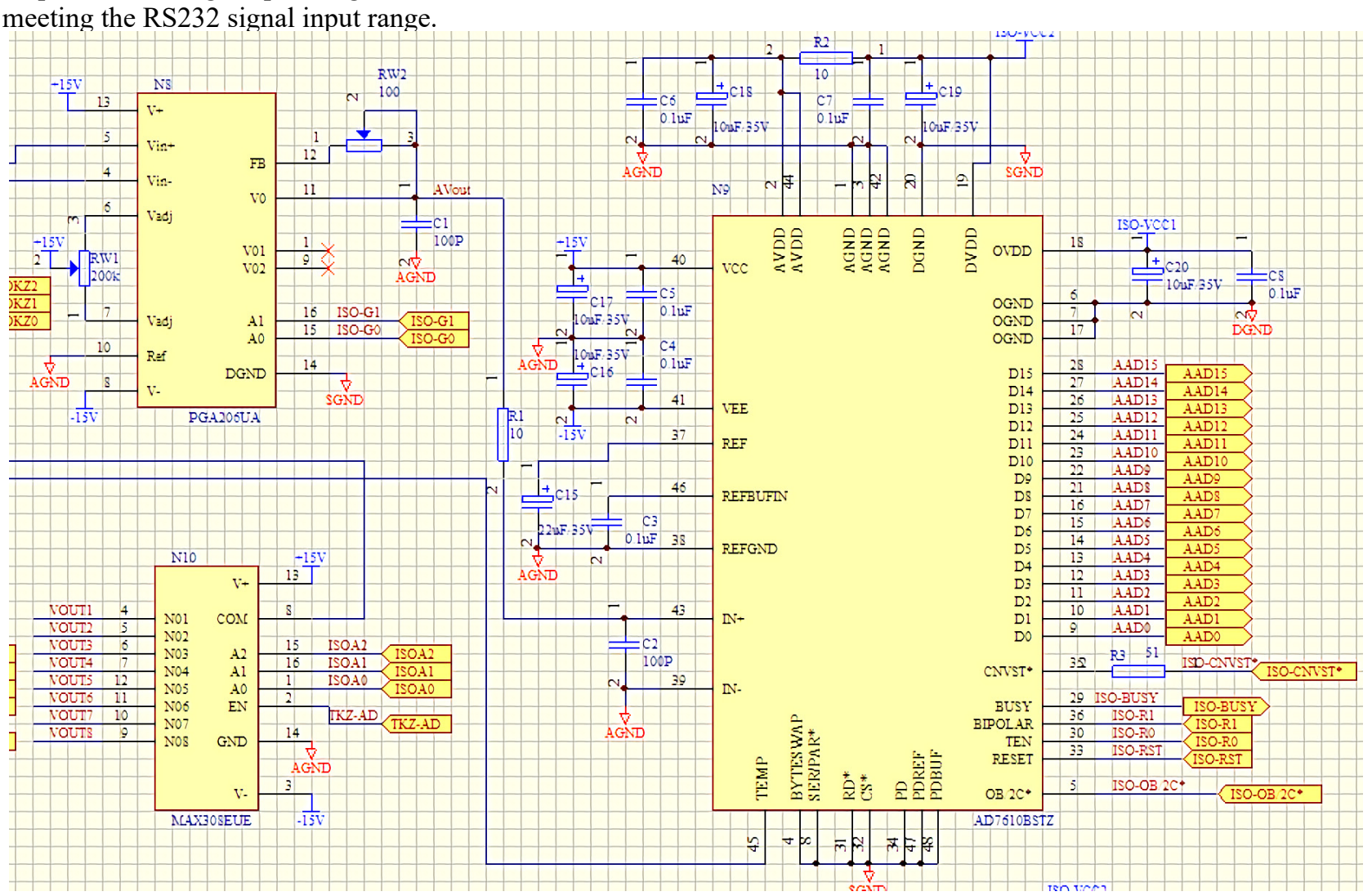

Fig. 4. Schematic diagram of $\mathrm{AD}$ converter interface.

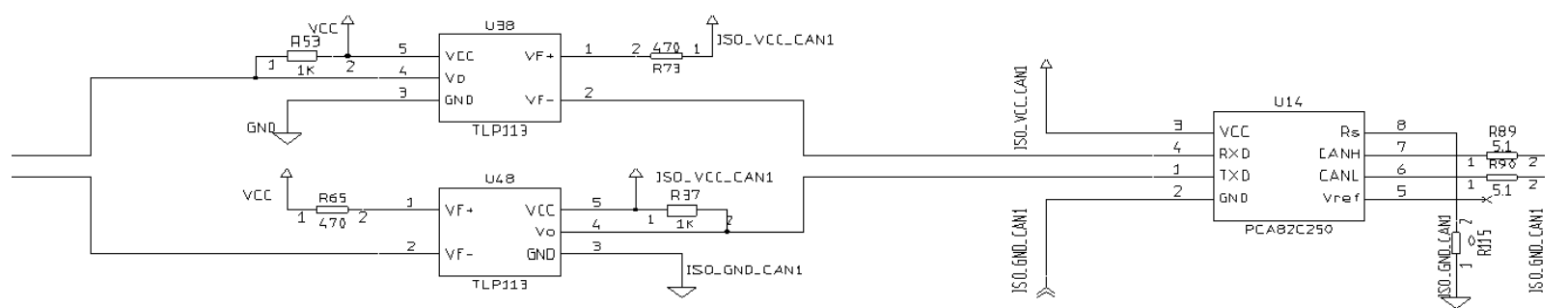

Fig. 5. Schematic diagram of CAN bus interface.

\section{Conclusion}

This board selects STM32 microcontroller as the control core, PT100 platinum resistance as the temperature sensor, which has the characteristics of high precision, low cost and strong anti-interference ability, meets the requirements of flight control computer for temperature acquisition, and has the function of 12 channel servo serial port switching debugging, which has a broad application prospect.

\subsection{Isolated can bus interface circuit}

Can interface of STM32 is used in an bus, and pca82c250t chip is used to realize optocoupler isolation of CAN bus interface, shown in Figure 5. 
Journal of Nanjing University of Aerinautics \&Astronautics, Vol.37No.4.

4. X.Y., Dong, H.Y.Meng, L.B.Kong. (2018) BasedontheSTM32ThermistorPartialPressureType

Temperature Measuring System Design. Computer\& Digital Engineering, Vol.46No.4:846850 .
5. S.Li,X.G.Tuo, G.Y.Zhang, L.Chen.(2018)Design of multi channel temperature acquisition system based on stm32.Journal of Sichuan University of Science \& Engineering (Natural Science Edition), Vol. 31 No. 3:50-53. 